International Journal of Pure and Applied Mathematics

Volume 112 No. 2 2017, 285-291

ISSN: 1311-8080 (printed version); ISSN: 1314-3395 (on-line version)

url: http://www.ijpam.eu

doi: 10.12732/ijpam.v112i2.6

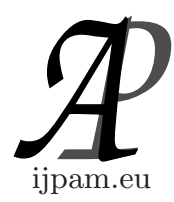

\title{
A NOTE ON AF-GROUPS AND FNS-GROUPS
}

\author{
Houshang Behravesh ${ }^{1}$, Shahram Torabikiya ${ }^{2} \S$ \\ ${ }^{1,2}$ Department of Mathematics \\ Faculty of Science \\ Urmia University, Urmia, IRAN
}

\begin{abstract}
The quantities $c(G), q(G)$ and $p(G)$ for finite groups were defined by H. Behravesh. In this paper, we will calculate these quantities for $L_{p^{n}}$, where $p$ is an odd prime, $n \in N, n \geq 3$.
\end{abstract}

AMS Subject Classification: 20K01, 20B05

Key Words: quasi-permutation representations, $p$-groups, FNS-p-groups, character theory

\section{Introduction}

Let $G$ be a finite $p$-group. $G$ is an FNS group, if for any normal subgroup $H$ of $G$, either $G^{\prime} \leq H$ or $H \leq Z(G)$. For instance if $p$ be a prime and $G$ be a nonabelian group of order $p^{3}$ or $p^{4}$, then $G$ is an FNS-group, [8, Proposition 2.1].

A nonabelian $p$-group $G$ is called AF-group(Abelian Factor) if every proper quotient of $G$ is abelian. For example the groups $D_{8}$ and $Q_{8}$ are AF-groups.

By [8, Proposition 2.2], every AF-group is FNS-group. It is known that a group $H$ is called capable if there exists a group $G$ such that $H \cong G / Z(G)$.

By [8, Theorem 2.2] the only capable AF-p-groups are:

$\begin{array}{lrc}\text { Received: } & \text { August 30, } 2016 & \text { (C) } 2017 \text { Academic Publications, Ltd. } \\ \text { Revised: } & \text { December 11, 2016 } & \text { url: www.acadpubl.eu } \\ \text { Published: } & \text { February 1, 2017 }\end{array}$

${ }^{\S}$ Correspondence author 
a) $D_{8}$ if $p=2$.

b) $L_{p^{3}}$ if $p \neq 2$. That is:

$$
\begin{aligned}
L_{p^{n}}=<a, b, c: a^{p^{n-2}}=b^{p}=c^{p}=[a, b]=[a, c] & =1, \\
{[b, c] } & =a^{p^{n-3}}>, \quad n \in N, n \geq 3 .
\end{aligned}
$$

If $G$ is a finite linear group of degree $n$, that is, a finite group of automorphisms of an $n$-dimensional complex vector space, we shall say that $G$ is quasi-permutation group if the trace of every element of $G$ has a non-negative rational integer. The trace of the automorphism corresponding to an element $x$ of $G$ equal to the number of letters left fixed by $x$, and so is non-negative integer. Thus, a permutation group of degree $n$ has a representation as a quasipermutation group of degree $n$. See [10].

By a quasi-permutation matrix we mean a square matrix over the complex field $C$ with non-negative integral trace. Thus every permutation matrix over $C$ is a quasi-permutation matrix. For a given finite group $G$, let $p(G)$ denote the minimal degree of a faithful permutation representation of $G$, let $q(G)$ denote the minimal degree of a faithful representation of $G$ by quasi-permutation matrices over the rational field $Q$, and let $c(G)$ be the minimal degree of a faithful representation of $G$ by complex quasi-permutation matrices. See [3].

It is easy to see $c(G) \leq q(G) \leq p(G)$, where $G$ is a finite group.

Now we want to calculating $c(G), q(G)$ and $p(G)$, for capable AF-p-groups and in patricular for $L_{p^{n}}, n \geq 4$ and the central product of two groups $L_{p^{n}}$, where $p$ is an odd prime, $n \in N, n \geq 3$.

\section{Quasi-Permutation Representation of $L_{p^{n}}$}

In this section we want to calculating quasi-permutation representation of $L_{p^{n}}$ and the central product of two groups $L_{p^{n}}$, where $p$ is an odd prime, $n \in N, n \geq$ 3 .

Theorem 1. Let $G$ be a finite $p$-group and $\left|G^{\prime}\right|=p$. Then $G$ is FNS-pgroup.

Proof. Let $H$ be a normal subgroup in $G$. Since $G$ has class 2 , then $G^{\prime} \leq$ $Z(G)$ and $1 \leq[G, H] \leq G^{\prime}$. Therefore we have two possible case.

If $[G, H]=1$, then $H \leq Z(G)$.

If $[G, H]=G^{\prime}$ and since $H$ be a normal subgroup in $G$, then $[G, H] \leq H$ and therefore $G^{\prime} \leq H$. 
Theorem 2. Let $\left|G^{\prime}\right|=p$ a prime. Assume that $G^{\prime} \leq Z(G)$. Then $\chi(1)^{2}=$ $|G: Z(G)|$ for every non linear $\chi \in \operatorname{Irr}(\mathrm{G})$.

Proof. Let $\chi$ be a non-linear, irreducible character of $G$. From [7, Corollary 2.28], we have $Z(G)=\bigcap\{Z(\chi): \chi \in \operatorname{Irr}(\mathrm{G})\}$. So $G^{\prime} \leq Z(G) \leq Z(\chi), G / Z(\chi)$ is abelian. From [7, Theorem 2.31], we have $\chi(1)^{2}=|G: Z(\chi)|$. Since $\left|G^{\prime}\right|=p$, a prime, either $G^{\prime} \cap \operatorname{ker} \chi=1$ or $G^{\prime} \leq \operatorname{ker} \chi$. Suppose $G^{\prime} \leq \operatorname{ker} \chi$. Then $G / \operatorname{ker} \chi$ is abelian. By [7, Lemma 2.27(e)], $\chi=\chi(1) \lambda$ for some linear character $\lambda$ of $Z(\chi)$, contracting that $\chi$ is non-linear and irreducible character of $G$. Hence $G^{\prime} \cap \operatorname{ker} \chi=1$. We will show that $Z(\chi) \leq Z(G)$.

Suppose $z \in Z(\chi)$. By [7, Lemma 2.27(f)], we have $Z(\chi) / \operatorname{ker} \chi=Z(G / \operatorname{ker} \chi)$. So, for any $g \in G,[z, g] \in \operatorname{ker} \chi$, i.e $[z, g] \in \operatorname{ker} \chi \cap G^{\prime}=1$. Hence $z \in Z(G)$ and so $Z(\chi) \leq Z(G)$ and $\chi(1)^{2}=|G: Z(G)|$.

Theorem 3. [5, Theorem B] Let $G$ be a finite p-group such that $|G: Z(G)|$ be a square and we denote character degree of $G$ by $\mathrm{cd}(\mathrm{G})$. Then the following statements are equivalent:

a) $\operatorname{cd}(\mathrm{G})=\left\{1,|G: Z(G)|^{1 / 2}\right\}$.

b) For any normal subgroup $H$ of $G$, either $G^{\prime} \leq H$ or $H \leq Z(G)$.

Corollary 4. Let $G$ be a finite p-group such that $|G: Z(G)|$ be a square. Then $G$ is an FNS-p-group iff $\operatorname{cd}(\mathrm{G})=\left\{1,|G: Z(G)|^{1 / 2}\right\}$.

Proof. The result is immediate by Theorem 3 .

Corollary 5. Let $G$ be a finite $p$-group such that $G / Z(G) \cong Z_{p} \times Z_{p}$. Then $G$ is an FNS-p-group.

Proof. Since $G$ is a finite $p$-group and $G / Z(G) \cong Z_{p} \times Z_{p}$, therefore $G / Z(G)$ is abelian and $|G: Z(G)|=p^{2}$. By Corollary 4 , that is enough to prove $\mathrm{cd}(\mathrm{G})=$ $\{1, p\}$.

Since $\chi(1)^{2}|| G: Z(G) \mid$ and $|G: Z(G)|=p^{2}$ therefore $\chi(1)^{2} \mid p^{2}$. Then $|\chi(1)|=$ 1 or $|\chi(1)|=p$ and $\operatorname{cd}(\mathrm{G})=\{1, p\}$.

Theorem 6. [1, Theorem 4.12] Let $G$ be a finite p-group of class 2 and let $Z(G)$ be cyclic. Then $c(G)=|Z(G)| .|G: Z(G)|^{1 / 2}$. Moreover when $p \neq 2$ then $c(G)=q(G)=p(G)$.

Now we want to calculating $c(G), q(G)$ and $p(G)$, for capable AF-p-groups.

Lemma 1. Let $G$ be capable AF-p-group. Then By [8, Theorem 2.2], G is of the form:

a) $D_{8}$ if $p=2$, 
b) $L_{p^{3}}$ if $p \neq 2$,

in this case we have $c\left(D_{8}\right)=4, c\left(L_{p^{3}}\right)=p^{2}$.

Proof. Let $G=L_{p^{3}}$. Then

$$
\begin{gathered}
G=<a, b, c: a^{p}=b^{p}=c^{p}=[a, b]=[a, c]=1, \\
{[b, c]=a>, Z(G)=<a>=G^{\prime},}
\end{gathered}
$$

and $|G: Z(G)|=p^{2}$. So $G / Z(G)$ is abelian and $G^{\prime} \leq Z(G)$. Hence $G$ has class 2 , by Theorem 6 ,

$$
c(G)=q(G)=p(G)=|Z(G)| \cdot|G: Z(G)|^{1 / 2}=p^{2} .
$$

If $G=D_{8}$, then by [2, Remark (3), part (b)], $c(G)=q(G)=p(G)=4$.

Theorem 7. [4, Theorem 2] Let $G$ be a nilpotent group and $G=H \times K$. Then $p(G)=p(H)+p(K)$.

Theorem 8. Let $G$ be a finite $p$-group of the form $L_{p^{n}}$. Then a) $G / G^{\prime} \cong C_{p^{n-3}} \times C_{p} \times C_{p},|Z(G)|=p^{n-2}$,

b) The number of nonlinear irreducible character of $L_{p^{n}}$ equal to $\phi(|Z(G)|)$, c) $c(G)=q(G)=p(G)=p^{n-1}$.

Proof. a) Let

$$
G=L_{p^{n}}=<a, b, c: a^{p^{n-2}}=b^{p}=c^{p}=[a, b]=[a, c]=1,[b, c]=a^{p^{n-3}}>.
$$

Then we have:

$G^{\prime}=<a^{p^{n-3}}>,\left|G^{\prime}\right|=p,\left|G: G^{\prime}\right|=p^{n-1}, Z(G)=<a>\cong C_{p^{n-2}},|Z(G)|=p^{n-2}$.

Furthemore since

$$
G / G^{\prime}=<a G^{\prime}, b G^{\prime}, c G^{\prime}>,\left(b G^{\prime}\right)^{p}=G^{\prime},\left(c G^{\prime}\right)^{p}=G^{\prime},\left(a G^{\prime}\right)^{p^{n-3}}=a^{p^{n-3}} G^{\prime}=G^{\prime}
$$

so

$$
\begin{gathered}
G / G^{\prime}=<a, b, c: a^{p^{n-3}}=b^{p}=c^{p}=[a, b]=[a, c]=1,[b, c]=a^{p^{n-3}}>, \\
G / G^{\prime} \cong C_{p^{n-3}} \times C_{p} \times C_{p} .
\end{gathered}
$$


b) Since

$$
|G|=\left|G: G^{\prime}\right|+\sum_{\chi \in \operatorname{Irr}(\mathrm{G}), \chi(1)>1} \chi(1)^{2}
$$

so

$$
p^{n}=p^{n-1}+\alpha \cdot p^{2} \Rightarrow \alpha=p^{n-2}-p^{n-3}=p^{n-2}(1-1 / p)=\phi\left(p^{n-2}\right)=\phi(|Z(G)|)
$$

therefore the number of nonlinear irreducible character of $L_{p^{n}}$ equal to $\phi(|Z(G)|)$.

c) Since $|G / Z(G)|=p^{2}$, so $G / Z(G)$ is abelian and $G^{\prime} \leq Z(G)$. Hence $G$ has class 2 , by Theorem 6 ,

$$
c(G)=q(G)=p(G)=|Z(G)| \cdot|G: Z(G)|^{1 / 2}=p^{n-1} .
$$

Notation. We denote the central product of groups $H, G$ by $H * G$.

Lemma 2. Let $H$ be the central product of 2 copies of the group $L_{p^{n}}$, with $n \geq 3$. Then:

a) For $i, j \in\{1,2\}$ we have:

$$
\begin{gathered}
H=<a, b_{1}, b_{2}, c_{1}, c_{2}: a^{p^{n-2}}=b_{i}{ }^{p}=c_{i}{ }^{p}=1, \\
{\left[a, b_{i}\right]=\left[a, c_{i}\right]=\left[b_{i}, b_{j}\right]=\left[c_{i}, c_{j}\right]=1,\left[b_{i}, c_{j}\right]=a^{\delta i j \cdot p^{n-3}}>.}
\end{gathered}
$$

b) $c(H)=q(H)=p(H)=p^{n}$.

Proof. a) By an easy calculation and by definition of group $L_{p^{n}}$, the first part is obviously.

b) Since $H=L_{p^{n}} * L_{p^{n}}$, then $H^{\prime}=<a^{p^{n-3}}>$ and $Z(H)=<a>\cong C_{p^{n-2}}$ and so $H^{\prime} \leq Z(H)$. Therefore by Theorem 6 ,

$$
c(H)=q(H)=p(H)=|Z(H)| \cdot|H: Z(H)|^{1 / 2}=p^{n-2} p^{2}=p^{n} .
$$

Theorem 9. Let $H$ be the central product of $r$ copies $(r \geq 1)$ of the group $L_{p^{n}}$, with $n \geq 3$. Then

a) For $i, j \in\{1,2, \ldots, r\}$ we have:

$$
\begin{gathered}
H=<a, b_{1}, b_{2}, \ldots, b_{r}, c_{1}, c_{2}, \ldots, c_{r}: a^{p^{n-2}}=b_{i}^{p}=c_{i}^{p}=1, \\
{\left[a, b_{i}\right]=\left[a, c_{i}\right]=\left[b_{i}, b_{j}\right]=\left[c_{i}, c_{j}\right]=1,\left[b_{i}, c_{j}\right]=a^{\delta i j \cdot p^{n-3}}>,} \\
\text { b) } c(H)=p(H)=q(H)=p^{n+r-2} .
\end{gathered}
$$


Proof. a) By definition of group $L_{p^{n}}$ we have :

$$
\begin{aligned}
& a_{1}^{p^{n-2}}=b_{1}{ }^{p}=c_{1}{ }^{p}=\left[a_{1}, b_{1}\right]=\left[a_{1}, c_{1}\right]=1,\left[b_{1}, c_{1}\right]=a_{1} p^{p^{n-3}}, \\
& a_{2}{ }^{p^{n-2}}=b_{2}{ }^{p}=c_{2}{ }^{p}=\left[a_{2}, b_{2}\right]=\left[a_{2}, c_{2}\right]=1,\left[b_{2}, c_{2}\right]=a_{2} p^{p^{n-3}}, \\
& a_{r}{ }^{p^{n-2}}=b_{r}{ }^{p}=c_{r}{ }^{p}=\left[a_{r}, b_{r}\right]=\left[a_{r}, c_{r}\right]=1,\left[b_{r}, c_{r}\right]=a_{r}{ }^{p^{n-3}} .
\end{aligned}
$$

Then

$$
a^{p^{n-2}}=b_{i}^{p}=c_{i}^{p}=\left[a, b_{i}\right]=\left[a, c_{i}\right]=1,\left[b_{i}, c_{i}\right]=a^{p^{n-3}} .
$$

By the second property of $\left[4\right.$, Definition 19.3], we have $\left[b_{i}, b_{j}\right]=\left[c_{i}, c_{j}\right]=$ $1,\left[b_{i}, c_{j}\right]=1$ and therefore

$$
\begin{gathered}
H=<a, b_{1}, b_{2}, \ldots, b_{r}, c_{1}, c_{2}, \ldots, c_{r}: a^{p^{n-2}}=b_{i}^{p}=c_{i}^{p}=1, \\
{\left[a, b_{i}\right]=\left[a, c_{i}\right]=\left[b_{i}, b_{j}\right]=\left[c_{i}, c_{j}\right]=1,\left[b_{i}, c_{j}\right]=a^{\delta i j \cdot p^{n-3}}>}
\end{gathered}
$$

where $i, j \in\{1,2, \ldots, r\}$.

b) By structure of group $H$, we have:

$$
Z(H)=<a>, H^{\prime}=<a^{p^{n-3}}>.
$$

Then by Theorem 6 ,

$$
c(H)=p(H)=q(H)=|Z(H)| \cdot|H: Z(H)|^{1 / 2}=p^{n-2} \cdot p^{r}=p^{n+r-2} .
$$

\section{References}

[1] H. Behravesh, Quasi-permutation representations of p-groups of class 2, J. London Math. Soc., 55, No. 2 (1997), 251-260.

[2] H. Behravesh, Characters and quasi-permutation representations of nilpotent groups of class 2 with two character degrees, Inter. Math. J., 4, No. 2 (2003), 137-143.

[3] J.M. Burns, B. Goldsmith, B. Hartley, R. Sandling, On quasi-permutation representations of finite groups, Glasgow Math. J., 36 (1994), 301-308.

[4] K. Dorek, T. Hawkes, Finite Suluble Groups, Walter de Gruyter, Berlin-New Yourk, 1992.

[5] G.A. Fernandez-Alcober, A. Moret, Groups with two extrem characters degrees and their normal subgroups, Trans of the American Math. Sve., 353, No. 6 (2001), 2171-2192.

[6] D. Gorenstein, Finite Groups, Harper-Row, New York-Evanston-Lonon, 1968.

[7] I.M. Isaacs, Character Theory of Finite Groups, Academic Press, New York, 1976. 
[8] G. Silberberg, Finite p-groups with few normal subgroups, Bull. Belg. Math. Soc., 7 (2000), 365-376.

[9] D. Wright, Degree of minimal embeddings for some direct products, American Journal of Mathematics, 91, No. 4 (1975), 897-903.

[10] D.J. Wong, Linear groups analogous to permutation groups, J. Austral. Math. Soc., A3 (1963), 180-184. 
